Journal of Computer Science 8 (7): 1085-1091, 2012

ISSN 1549-3636

(C) 2012 Science Publications

\title{
Artificial Neural Network Based Rotor Capacitive Reactance Control for Energy Efficient Wound Rotor Induction Motor
}

\author{
${ }^{1}$ Ranjith Kumar, K., ${ }^{2}$ S. Palaniswami and ${ }^{1}$ K. Siva Kumar \\ ${ }^{1}$ Department of Electrical Engineering, \\ Government College of Technology, Coimbatore, India \\ ${ }^{2}$ Government College of Engineering, Bargur, Krishnagiri, India
}

\begin{abstract}
Problem statement: The Rotor reactance control by inclusion of external capacitance in the rotor circuit has been in recent research for improving the performances of Wound Rotor Induction Motor (WRIM). The rotor capacitive reactance is adjusted such that for any desired load torque the efficiency of the WRIM is maximized. The rotor external capacitance can be controlled using a dynamic capacitor in which the duty ratio is varied for emulating the capacitance value. This study presents a novel technique for tracking maximum efficiency point in the entire operating range of WRIM using Artificial Neural Network (ANN). The data for ANN training were obtained on a three phase WRIM with dynamic capacitor control and rotor short circuit at different speed and load torque values. Approach: A novel neural network model based on the back-propagation algorithm has been developed and trained in determining the maximum efficiency of the motor with no prior knowledge of the machine parameters. The input variables to the ANN are stator current $\left(\mathrm{I}_{\mathrm{s}}\right)$, Speed $(\mathrm{N})$ and Torque $\left(\mathrm{T}_{\mathrm{m}}\right)$ and the output variable is the duty ratio (D). Results: The target is pre-set and the accuracy of the ANN model is measured using Mean Square Error (MSE) and $\mathrm{R}^{2}$ parameters. The result of $\mathrm{R}^{2}$ value of the proposed ANN model is found to be 0.99980 . Conclusion: The optimal duty ratio and corresponding optimal rotor capacitance for improving the performances of the motor are predicted for low, medium and full loads by using proposed ANN model.
\end{abstract}

Key words: Artificial Neural Network (ANN), Wound Rotor Induction Motor (WRIM), Torque ( $\mathrm{T}_{\mathrm{m}}$ ), Digital Signal Processor (DSP), rotor reactance control, corresponding optimal rotor

\section{INTRODUCTION}

It is known from the literature and energy saving points more than $60 \%$ of total electrical energy consumed worldwide is by Induction motors. Although Induction motors, specifically, squirrel cage types (Sarhan and Issa, 2006) are widely used in electrical drives, Wound Rotor Induction Motor (WRIM) has some distinct applications like high torque, starting with high inertia loads, adjustable speed drives and soft starts. Hence the utility of wound rotor induction motor has also been increased on par with squirrel cage types of motors.

The primary research focus on these motors is to improve the efficiency for significantly saving electrical energy. In the literature, several rotor reactance control schemes have been proposed to control the speed, torque and performance of the motor. A novel method for controlling the speed of WRIM by neutralizing the rotor inductive reactance with the help of rotor externally connected capacitive reactance have also been dealt in the literature. In this method, the motor develops maximum torque. The rotor resonant is obtained by introducing a suitable capacitive reactance to cancel the rotor winding reactance for each slip frequency condition. The main drawback of this method is the requirement of high value of capacitance (order of a Farad) required to operate the machine closes to rotor resonance conditions.

In order to overcome this problem, a switched capacitor concept has been adopted for the rotor reactance control of an induction motor to improve performance parameters. It utilizes the concept of switched capacitor in which it makes use of four Thyristors as switches to form an $\mathrm{H}$ - bridge circuit and a single capacitor. The complementary switches are fired using Pulse Width Modulation (PWM) pulses. The improvement of various performance parameters and speed control of the WRIM were presented by this method. Another technique in which the speed control

Corresponding Author: Ranjith Kumar, K., Department of Electrical Engineering, Government College of Technology, Coimbatore, India Tel: +919443015058 
of WRIM is obtained using chopper controlled external resistance enhanced with a dc capacitor. The efficiency of the motor is significantly reduced due to the external rotor resist or control. Sivanandam and Deepa (2007) have described the various soft computing techniques.

The different ANN techniques and its applications to solve complex and non-linear functions of the induction motor and AC systems are presented (Benslimane et al., 2006; Krishan et al., 2010). Ranjithkumar and Palaniswami (2011) have presented a fuzzy controller based rotor capacitive reactance control under rotor resonant conditions for enhancing the performance of the wound rotor induction motor.

This study proposes two novel techniques to control WRIM and operate the motor closes to maximum efficiency. The two methods are named as indirect rotor capacitive reactance control scheme and ANN based control scheme. In the first scheme, the rotor circuit is inserted with a dynamic capacitor to vary the capacitance dynamically. The duty ratio is adjusted for emulating the capacitance value dynamically with respect to load variables. A relationship derived between stator current for different loads and duty ratio required to control the dynamic capacitor for maximum efficiency point of the drive has been established.

In the second scheme, the optimal duty ratio is made as a function of stator current, speed and torque. A feed forward network is trained to relate this complex and nonlinear function.

\section{MATERIALS AND METHODS}

Indirect Rotor capacitive reactance control scheme: Dynamic switched capacitor concept: A dynamic switched capacitor consists of a capacitor and $\mathrm{H}$ bridge circuit with bi-directional switch pairs as shown in Fig. 1 and 2. The switch pairs (S1, S4) and (S2, S3), respectively, are fired using pulses generated using DSP controller. During the time interval, when the switches $(\mathrm{S} 1, \mathrm{~S} 4)$ are $\mathrm{ON}$ the capacitor is charging and a serial $\mathrm{R}_{\mathrm{r}}-$ $\mathrm{L}_{\mathrm{r}}-\mathrm{C}_{\mathrm{r}}$ circuit is formed.

In the time interval, when the switches $(\mathrm{S} 2, \mathrm{~S} 3)$ are $\mathrm{ON}$ the capacitor is applied with reverse polarity to the $\mathrm{R}_{\mathrm{r}}-\mathrm{L}_{\mathrm{r}}$ circuit and the capacitor starts discharging. The time period and duty ratio are given in Eq. 1 and 2 respectively:

$\mathrm{T}=\mathrm{T}_{\mathrm{on}}+\mathrm{T}_{\text {off }}$

$\mathrm{d}=\frac{\text { Ton }}{\mathrm{T}}$

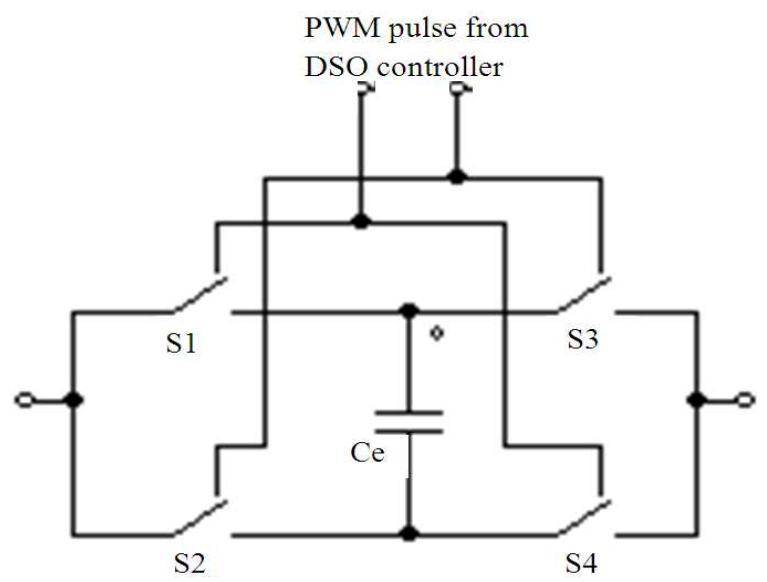

Fig. 1: Dynamic Capacitor circuit

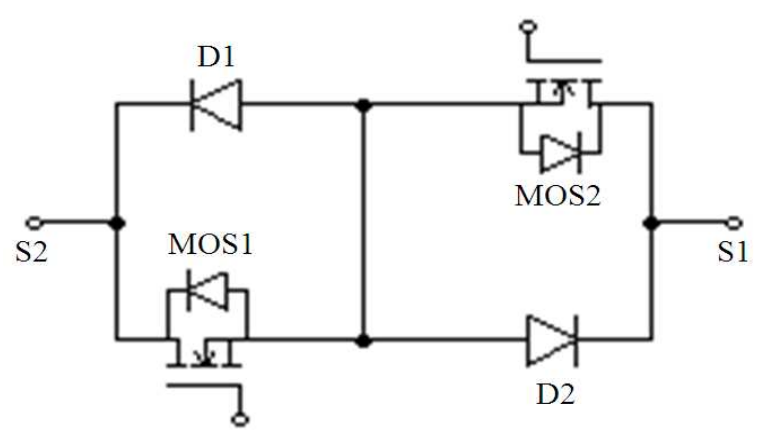

Fig. 2: Bidirectional switch $\mathrm{S}_{1}$

Thereby, the resultant effective value of the rotor capacitance is given in Eq. 3:

$\mathrm{Ce}=\frac{\mathrm{C}}{(2 \mathrm{~d}-1)^{2}}$

The dynamic capacitor concept is used in the proposed method to obtain the capacitance required for controlling in the rotor circuit. The effective value of capacitance with respect to the duty ratio for $\mathrm{C}=100 \mu \mathrm{F}$ is shown in Fig. 3.

Proposed indirect rotor capacitive reactance control scheme: A three phase wound rotor machine with rating and parameters shown in Table 1 is used for experimental setup. The power circuit configuration of the rotor capacitive control scheme is shown in Fig. 4. The capacitor used for the $\mathrm{H}$ - bridge circuit was $100 \mu \mathrm{F}$ and the switching frequency was about $4 \mathrm{kHz}$. 


\section{J. Computer Sci., 8 (7): 1085-1091, 2012}

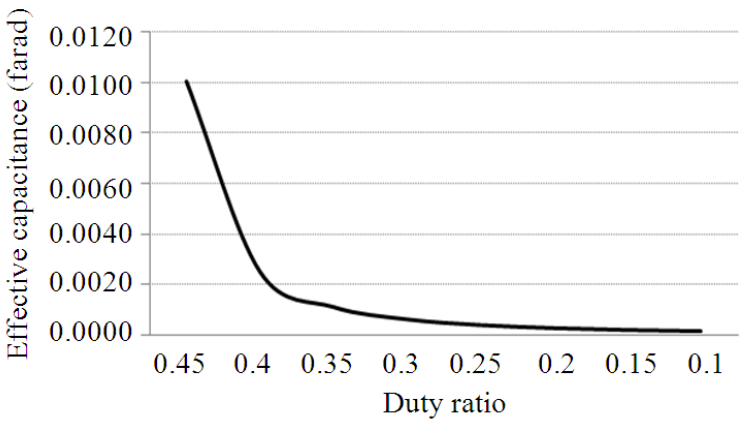

Fig. 3: Ce Vs Duty ratio for $\mathrm{C}=100 \mu \mathrm{F}$

Table 1: Specifications for the simulation and experiment

\begin{tabular}{lll}
\hline Components & Part names/ Manufacturer & Rating values \\
\hline $\mathrm{P}, \mathrm{V}$ & $\begin{array}{l}\text { Motor rating } \\
\text { (Power, Voltage) } \\
\text { (frequency, } \\
\text { Poles) }\end{array}$ & $2.2 \mathrm{~kW}-415 \mathrm{~V}$ \\
$\mathrm{Rs}$ & Stator resistance & $50 \mathrm{~Hz}-4$ \\
$\mathrm{Rr}$ & Rotor resistance & $5.6 \mathrm{~W}$ \\
$\mathrm{Ls}, \mathrm{Lr}$ & $\begin{array}{l}\text { Stator and rotor inductance } \\
\mathrm{Lm}\end{array}$ & $2.22 \mathrm{~W}$ \\
\hline
\end{tabular}

Table 2: $\mathrm{R}^{2}$ and Mean Square Error value

\begin{tabular}{lll}
\hline No of nodes in hidden layer & MSE & $\mathrm{R}^{2}$ \\
\hline 1 & 0.161 & 0.96373 \\
2 & 0.935 & 0.99820 \\
3 & 0.048 & 0.99980 \\
4 & 0.262 & 0.99920 \\
5 & 1.220 & 0.99920 \\
6 & 2.610 & 0.99940 \\
7 & 2.210 & 0.99920 \\
8 & 0.211 & 0.99940 \\
9 & 3.960 & 0.99960 \\
10 & 2.210 & 0.99960 \\
\hline
\end{tabular}

A TMS320F2407 Digital Signal Processor (DSP) controller was used for generating PWM pulses for appropriate switches. The pulses from DSP are given to switches through Opto-coupler for isolating the control and power circuits. The experimental setup has been given in Fig. 5. A power spectrum analyzer was used to measure the active, reactive power readings, stator voltage and current values.

The relations between the optimal efficiency, efficiency at rotor shorted, duty ratio for optimal efficiency and stator current are shown in Fig. 6. The experiment is repeated to ensure the relationship shown in Fig. 6.

\section{The following tests were performed:}

Variable torque with fixed capacitor: In the first instance, the tests were conducted for obtaining the efficiency, variation of speed at different loads with varying duty ratio.

Constant load with variable capacitor: In this case, the variation of performance parameters as function of the duty ratio for different loading conditions were analyzed.

A neural network model for optimal performance of WRIM: The experimentally derived relationship is nonlinear and need more memory to store the information in a digital computer.

ANN with many layers of neurons which is analogous to the biological neurons has the merits of speedy computational capability, can be used to approximate complicated nonlinear functions (Krishan et al., 2010).

In addition to this, Neural network posses learning capability and generalization. Hence a multilayer feed forward ANN (MFF-ANN) is used for training with the stator current, speed and motor torque as input and duty ratio as desired output (Fig. 7).

The implemented MFF-ANN model with back propagation algorithm for training is illustrated in Fig. 8.

To train the proposed ANN for predicting the optimal efficiency point, initially, three inputs, three hidden and one output neuron (structure of 3-3-1) has been used. The inputs are stator current $\left(\mathrm{I}_{\mathrm{s}}\right)$, rotor speed $(\mathrm{N})$ and Motor torque $\left(\mathrm{T}_{\mathrm{m}}\right)$ and the desired output is duty ratio to control the dynamic capacitor. The transfer functions used are Tangent sigmoid for hidden layer and a linear function for output neuron. The data used for training were obtained from experimentally measured results. The input and output are normalized and the neural network is trained with LevenMarquardt algorithm (L-M). The algorithm is repeated several times to determine the optimal amount of nodes in the hidden layers. The optimal hidden layers are selected based on the MSE and $\mathrm{R}^{2}$ parameter values. Finally, three nodes in the hidden layer are chosen as best based on the $\mathrm{R}^{2}$ and MSE.

The value of $\mathrm{R}^{2}$ and MSE for various combinations of HLs are given in Table 2. The number of hidden layers based on the convergence rate of the M-L algorithm is given in Table 3. The momentum factors, learning rate and maximum epochs are selected as 0.3 and 0.9 and 3000 epochs respectively for simulation. The training is stopped when the network error has reached 0.00001 . Finally, based on the comparison of Table 2 and 3, the best MSE is obtained as 0.048. From this, it is inferred that 3-3-1 is the best possible neural network structure. 


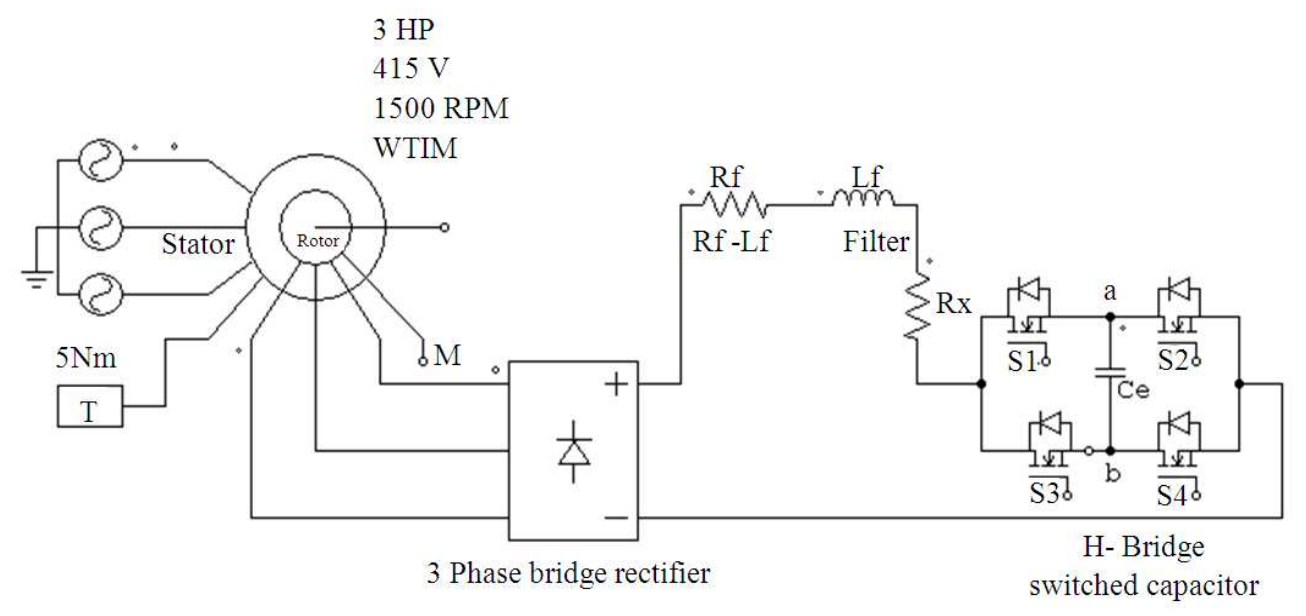

Fig. 4: Power circuit of Indirect Rotor capacitive recatance control scheme

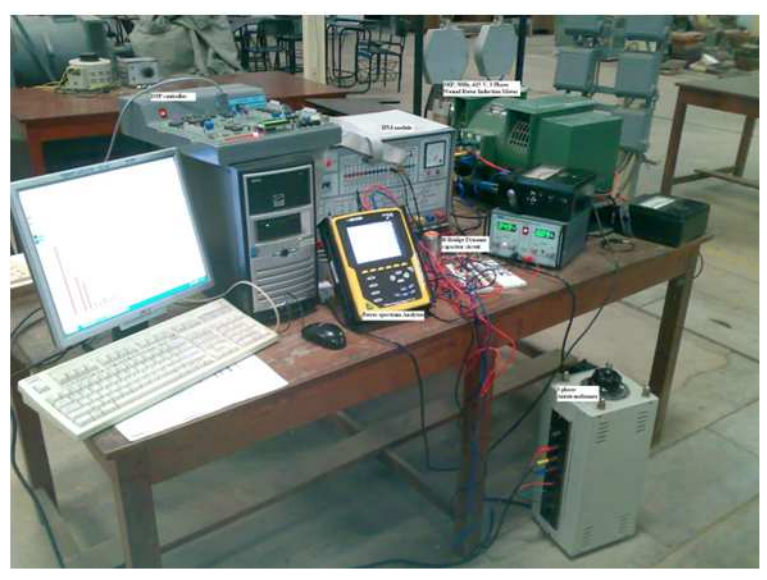

Fig. 5: Experimental setup

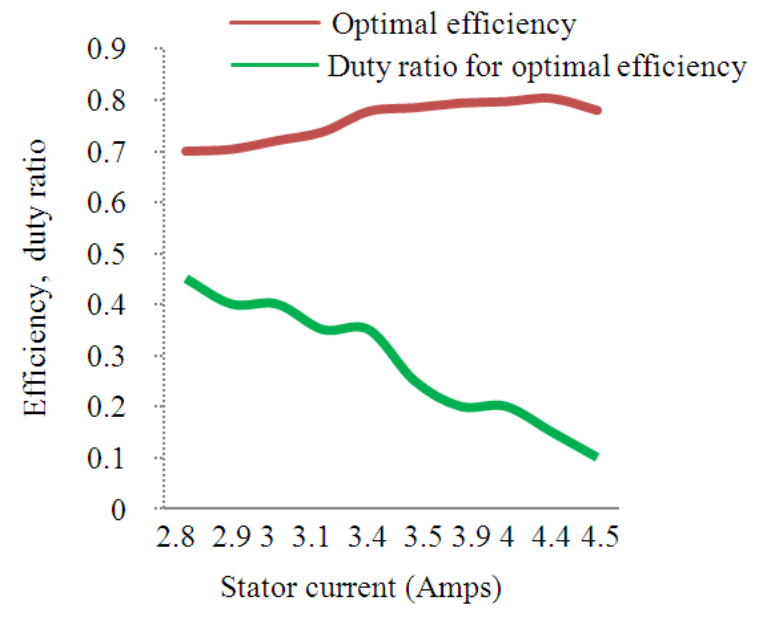

Fig. 6: Experimentally derived relationship between optimal efficiency and Duty ratio

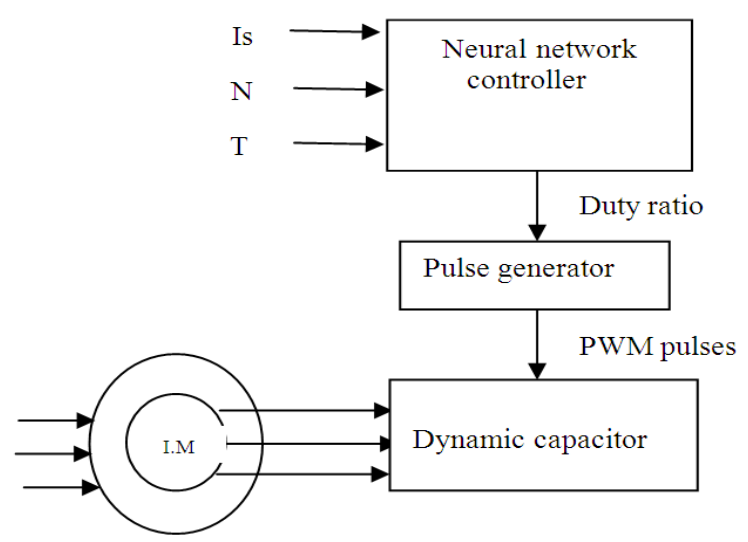

Fig. 7: Proposed Neural network controller

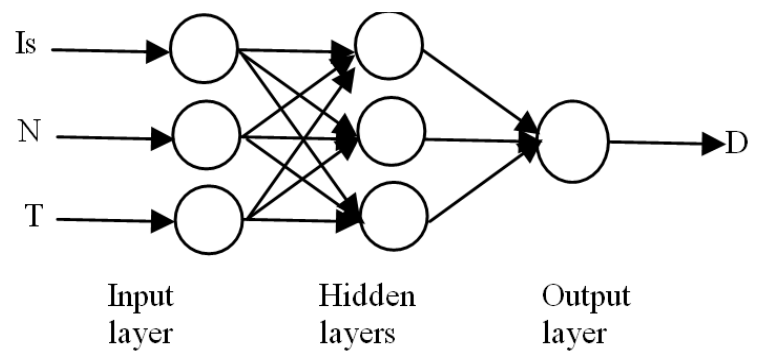

Fig. 8: Neural network model

Table 3: Effect of selection of hidden layers (HLs)

\begin{tabular}{llll}
\hline No of HLs & no of nodes in HL & MSE & $\mathrm{R}^{2}$ \\
\hline 1 & 3 & 0.048 & 0.9998 \\
2 & $3 / 3$ & 1.340 & 0.9992 \\
2 & $3 / 8$ & 0.158 & 0.9996 \\
3 & $3 / 3 / 3$ & 0.126 & 0.9994 \\
3 & $3 / 8 / 8$ & 1.120 & 0.9994 \\
3 & $3 / 8 / 11$ & 1.730 & 0.9992 \\
4 & $3 / 8 / 11 / 11$ & 1.380 & 0.9992 \\
\hline
\end{tabular}


J. Computer Sci., 8 (7): 1085-1091, 2012

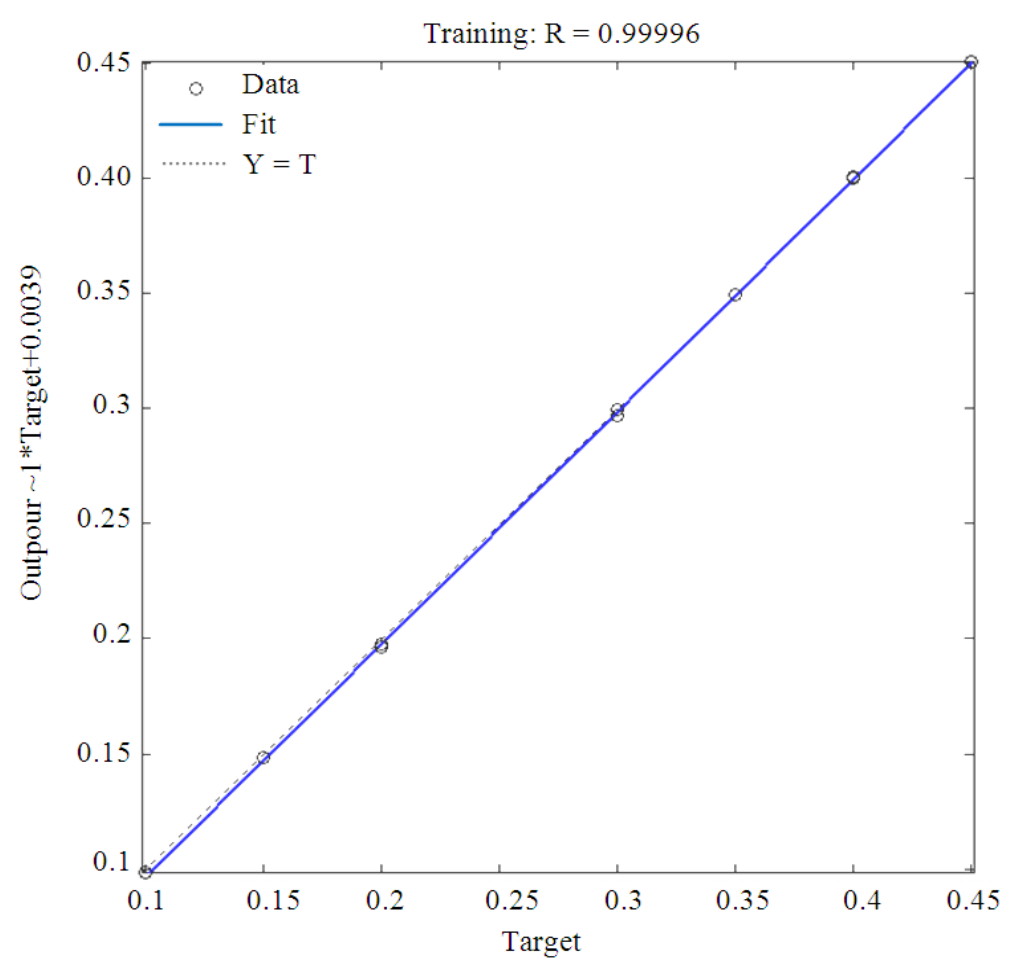

Fig. 9: Performance graph

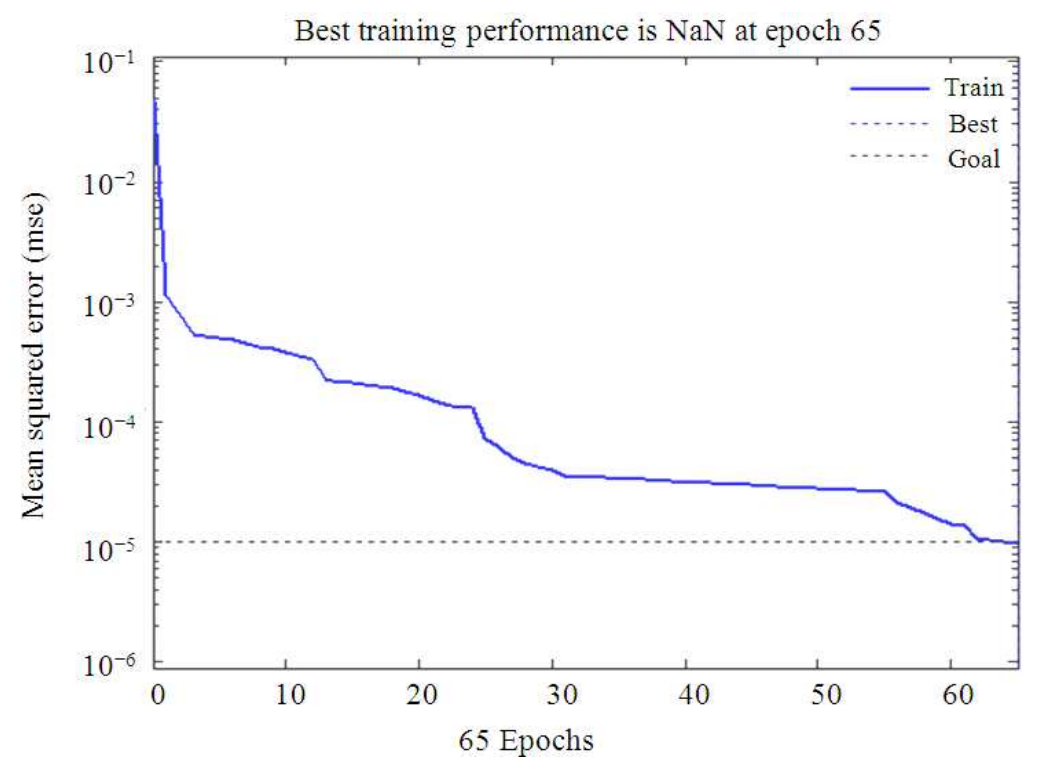

Fig. 10: Correlation between real and estimated values

The training is done in different combination of input data and the proposed network is tested for 10 sets of input data to reach the threshold value of 0.00001 . The goal is achieved in 65 epochs with error closes to 0.00001 as shown in Fig. 10. The target for duty ratio are trained as a light load, medium load and full load conditions. The comparison of test results, experimental results and the error difference are shown in Table 4. The optimal capacitance corresponding to the optimal duty ratio is calculated and shown in Table 4. 
J. Computer Sci., 8 (7): 1085-1091, 2012

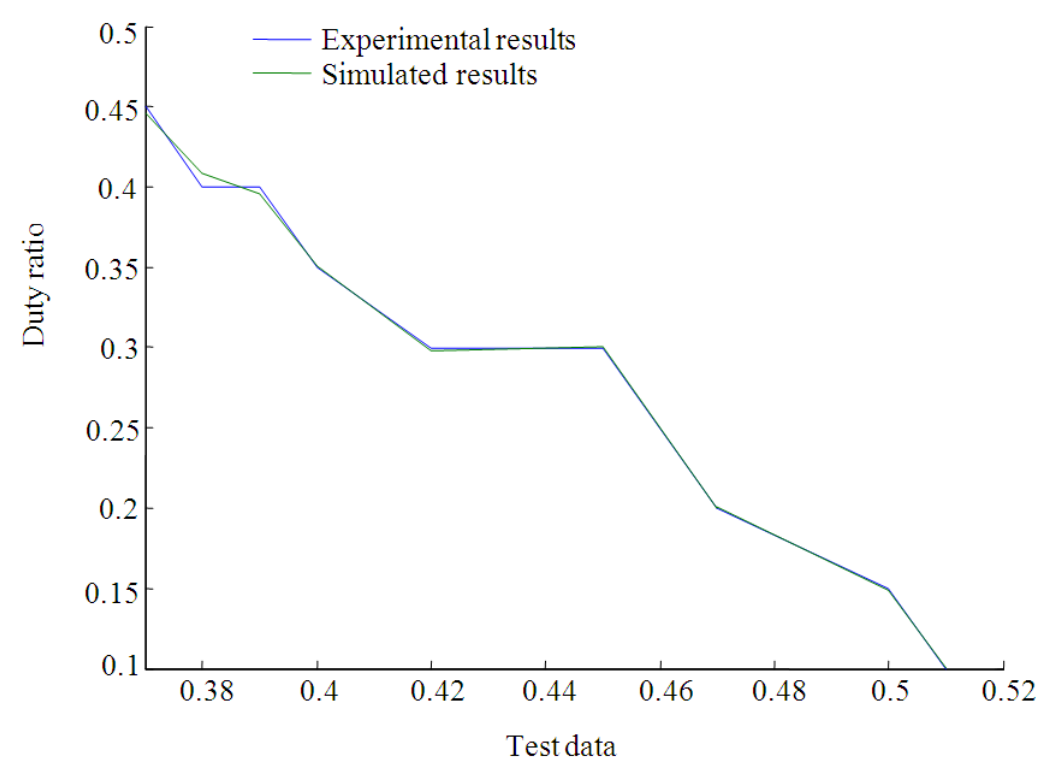

Fig. 11: Test results and simulated result

Table 4: Simulated results and Target

\begin{tabular}{|c|c|c|c|c|c|}
\hline \multirow[b]{2}{*}{ Load variation } & \multirow[b]{2}{*}{ Test } & \multirow[b]{2}{*}{ ANN } & \multirow[b]{2}{*}{ error difference } & \multicolumn{2}{|c|}{$\begin{array}{l}\text { Optimal capacitance } \\
\text { (In Farad) }\end{array}$} \\
\hline & & & & Test & ANN \\
\hline \multirow{3}{*}{$\begin{array}{l}\text { Light load } \\
(2.5 \mathrm{~A} \text { to } 3 \mathrm{~A})\end{array}$} & 0.45 & 0.4461 & 0.0039 & 0.01 & $8.61 \mathrm{E}-03$ \\
\hline & 0.40 & 0.4083 & -0.0083 & 0.0025 & 2.97E-03 \\
\hline & 0.40 & 0.3958 & 0.0042 & 0.0025 & $2.30 \mathrm{E}-03$ \\
\hline \multirow{4}{*}{$\begin{array}{l}\text { Medium load } \\
\text { (3.1 A to } 4 \mathrm{~A} \text { ) }\end{array}$} & 0.35 & 0.3505 & -0.0005 & $1.11 \mathrm{E}-03$ & $1.12 \mathrm{E}-03$ \\
\hline & 0.35 & 0.3508 & -0.0008 & $1.11 \mathrm{E}-03$ & $1.12 \mathrm{E}-03$ \\
\hline & 0.30 & 0.2981 & 0.0019 & $6.25 \mathrm{E}-04$ & $6.13 \mathrm{E}-04$ \\
\hline & 0.30 & 0.3000 & 0.0000 & $6.25 \mathrm{E}-04$ & $6.13 \mathrm{E}-04$ \\
\hline \multirow{4}{*}{$\begin{array}{l}\text { Full load } \\
\text { (4.1 to } 4.5 \mathrm{~A} \text { ) }\end{array}$} & 0.20 & 0.1998 & 0.0002 & $2.78 \mathrm{E}-04$ & $2.77 \mathrm{E}-04$ \\
\hline & 0.20 & 0.2011 & -0.0011 & $2.78 \mathrm{E}-04$ & $2.80 \mathrm{E}-04$ \\
\hline & 0.15 & 0.1493 & 0.0007 & 2.04E-04 & 2.03E-04 \\
\hline & 0.10 & 0.1001 & -0.0001 & $1.56 \mathrm{E}-04$ & $1.56 \mathrm{E}-04$ \\
\hline
\end{tabular}

\section{RESULTS AND DISCUSSION}

The performance graph, the correlation between real and estimated values are shown in Fig. 9 and 10 respectively. The test data used for simulation is selected randomly among the actually measured from experiment. The experiment and simulated ANN results shown in Fig. 11 for 10 test data. It is inferred that the proposed ANN has shown good agreement with actual measured data.

\section{CONCLUSION}

In this study, a novel indirect rotor capacitive reactance control scheme and a simple algorithm to predict the optimal rotor capacitance to run the motor on optimal efficiency point using artificial neural network are suggested. In the indirect control scheme, the duty ratio is adjusted to operate the motor to its maximum efficiency point for each load variation.

The data obtained were used to train the neural network model. The optimal duty ratio is computed as a function of stator current, rotor speed and motor torque to predict the optimal efficiency. The optimal rotor capacitance corresponding to the optimal efficiency point was also calculated for each load condition. It is inferred from the comparisons, the results of the proposed ANN technique show good agreement with the actual data.

The advantages of this method are accurate, fast estimation, insensitivity to parameter variations and load change. The proposed methods could be a better solution for energy efficient operation of WRIM used for high power applications.

\section{REFERENCES}

Benslimane, T., B. Chetate and R. Beguenane, 2006. Choice of input data type of artificial neural network to detect faults in alternative current systems. Am. J. Applied Sci., 3: 1979-1983. DOI: 10.3844/ajassp.2006.1979.1983

Krishan, M.M., L. Barazane and A. Khwaldeh, 2010. Using an adaptive fuzzy-logic system to optimize the performances and the reduction of chattering phenomenal in the control of induction motor. Am. J. Applied Sci., 7: 110-119. DOI: 10.3844/ajassp.2010.110.119 
Ranjithkumar, K. and S. Palaniswami, 2011. Performance enhancement of wound rotor induction motor by resonating the rotor circuit using fuzzy controller. Eur. J. Sci. Res., 52: 580-591.

Sarhan, H. and R. Issa, 2006. Improving mechanical characteristics of inverter-induction motor drive system. Am. J. Applied Sci., 3: 1961-1966. DOI: 10.3844/ajassp.2006.1961.1966
Sivanandam, S.N. and S.N. Deepa, 2007. Principles of Soft Computing. 1st Edn., Wiley India, New Delhi, ISBN-10: 8126510757, pp: 762. 